Subscriber access provided by Caltech Library

\title{
Quantum Electronic Structure
}

\section{Non-orthogonal configuration interaction with single substitutions for core-excited states: An extension to doublet radicals}

Katherine J. Oosterbaan, Alec F. White, and Martin Head-Gordon

J. Chem. Theory Comput., Just Accepted Manuscript • DOI: 10.1021/acs.jctc.8b01259 • Publication Date (Web): 24 Apr 2019

Downloaded from http://pubs.acs.org on April 24, 2019

\section{Just Accepted}

"Just Accepted" manuscripts have been peer-reviewed and accepted for publication. They are posted online prior to technical editing, formatting for publication and author proofing. The American Chemical Society provides "Just Accepted" as a service to the research community to expedite the dissemination of scientific material as soon as possible after acceptance. "Just Accepted" manuscripts appear in full in PDF format accompanied by an HTML abstract. "Just Accepted" manuscripts have been fully peer reviewed, but should not be considered the official version of record. They are citable by the Digital Object Identifier (DOI®). "Just Accepted" is an optional service offered to authors. Therefore, the "Just Accepted" Web site may not include all articles that will be published in the journal. After a manuscript is technically edited and formatted, it will be removed from the "Just Accepted" Web site and published as an ASAP article. Note that technical editing may introduce minor changes to the manuscript text and/or graphics which could affect content, and all legal disclaimers and ethical guidelines that apply to the journal pertain. ACS cannot be held responsible for errors or consequences arising from the use of information contained in these "Just Accepted" manuscripts. 


\title{
Non-orthogonal configuration interaction
} with single substitutions for core-excited states: An extension to doublet radicals

\author{
Katherine J. Oosterbaan, ${ }^{\dagger, \ddagger}$ Alec F. White, " and Martin Head-Gordon, ${ }^{*, \dagger}$ \\ $\dagger$ Department of Chemistry, University of California, Berkeley, California 94720, USA \\ $\ddagger$ Chemical Sciences Division, Lawrence Berkeley National Laboratory, Berkeley, California \\ 94720, USA \\ \Division of Chemistry and Chemical Engineering, California Institute of Technology, \\ Pasadena, California 91125, USA \\ E-mail: mhg@cchem.berkeley.edu
}

\begin{abstract}
In this paper we present an open-shell extension of the non-orthogonal configuration interaction singles (NOCIS) method for the calculation of core-excited states, intended for peak assignment in XAS spectra of doublet radicals. This extension requires the consideration of additional configurations due to the singly-occupied openshell orbital, and the addition of essential orbital relaxation effects is found to provide a significant improvement on standard CIS, while maintaining the desirable properties of spin-purity, variationality, and size-consistency. We apply this method to the calculation of core-excitations for several open-shell molecules and demonstrate that it performs competitively with other available methods, despite lack of dynamic correlation. In particular, relative to CVS-ADC(2)-x, RMS error is reduced by a factor of


6 over usual orthogonal CIS, and is comparable to time-dependent density functional theory with the best short-range corrected functionals.

\section{Introduction}

Open-shell molecules or free radicals, as they are sometimes known, are molecules of great interest in many areas of science. Not only do they play a key role in both normal and abnormal biological processes, ${ }^{1,2}$ but they also are involved in atmospheric chemistry processes such as soot ${ }^{3}$ formation, the interstellar chemistry of planetary ionospheres ${ }^{4-7}$ and, closer to home, in radiation-damaged systems. ${ }^{8,9}$ The study and characterization of these radicals is critical to furthering our understanding in almost every area of science.

One growing area in this study is that of x-ray absorption spectroscopy (XAS), which has the benefit of both environmental and element specificity. ${ }^{10,11}$ This technique is a relatively new and growing field, particularly as pertains to radical species. Specifically, the identification of characteristic peaks for smaller radical species is still underway, ${ }^{12,13}$ and is likely to be for some time. For example, studies that use NEXAFS to follow the effect of ionizing radiation on ice, as well as ices doped with organic molecules, ${ }^{14}$ often must hypothesize which molecules they are seeing at certain peaks. However, identification of these species, whose NEXAFS signature has not yet been characterized (e.g. the HCO radical in ref. 14), is particularly challenging. Thus, there is a great need for accurate theoretical methods that can be used to assist in the identification of these molecules' characteristic spectroscopic peaks.

To identify XAS peaks in new radical spectra, spectroscopists must turn to theoretical simulations of core excitations. From the electronic structure standpoint, it has long been known that radicals are far more challenging to treat than their closed-shell counterparts, for several reasons. ${ }^{15-19}$ One of the standard ways to determine ground states of molecules is unrestricted Hartree-Fock (UHF), but in radicals this method frequently fails to adequately 
predict even basic properties and reactivities. This is attributed to symmetry breaking problems, ${ }^{20,21}$ which often manifest as a high degree of spin-contamination: UHF wavefunctions are not eigenvalues of $\hat{S}^{2}$.

There have been several suggested work-arounds for these problems. One option, which we choose in this paper, is to use restricted open-shell Hartree-Fock (ROHF) instead of UHF. ROHF is already more desirable for the identification of XAS peaks, which are by nature spin-dependent, so it is an added benefit that this also helps eliminate some of the perils of working with radicals. However, it is also worth noting that ROHF is not a cureall; it has still been reported to fail in some situations, which was attributed to symmetry breaking problems and orbital instabilities. ${ }^{16}$ Additionally, ROHF wavefunctions are harder to converge, and ROHF theory does not permit bond-breaking.

It has also been reported that the inclusion of a higher level of electron correlation has helped to resolve some of these problems. Thus, methods like Kohn-Sham density functional theory (KS-DFT) have been found to be fairly successful at treating radicals. ${ }^{15}$ However, they suffer from the traditional problems of DFT methods, namely self-interaction errors as well as the inability to treat long-range effects such as charge transfer. ${ }^{22,23}$

There have also been a few other attempts to treat this problem, such as Brueckner coupled-cluster doubles, ${ }^{24}$ orbital-optimized coupled-cluster doubles ${ }^{25}$ and several MP2based methods. ${ }^{15} \mathrm{~A}$ final challenge to theoretical simulations of radicals, and of greatest interest to this paper, is that radicals and excitations from radical ground states tend to be multiconfigurational, meaning that a method that does not enable treatment of these cases can never hope to be sufficiently accurate.

Currently, the common methods used for core excitations are time-dependent density functional theory (TDDFT), ${ }^{26-31} \Delta$-SCF and $\Delta$-DFT, ${ }^{32}$ equation of motion coupled-cluster methods, ${ }^{33-39}$ algebraic diagrammatic construction (ADC) methods, ${ }^{40-45}$ static exchange (STEX), ${ }^{46}$ and configuration interaction singles (CIS). ${ }^{47,48}$ These methods have also been used for the calculation of open-shell radicals. ${ }^{49-53}$ The general benefits and pitfalls of all 
these methods were discussed in our previous work, ${ }^{54}$ so we will only touch on them in the results section as is relevant. It is worth noting, however, that several of them employ UHF rather than ROHF, leaving them susceptible to the problems discussed above. Lately, some other options that have been discussed are the selection of orbitals in CIS, ${ }^{55}$ a combination DFT $/ \Delta$-SCF method, ${ }^{56}$ and a core-valence-separation-based approach to coupled cluster. ${ }^{53}$

Recently, we introduced the non-orthogonal configuration interaction singles (NOCIS) method for the calculation of closed-shell core-excited states, ${ }^{54}$ a spin-pure, non-orthogonal generalization of CIS for core-excited states. This method includes orbital relaxation effects, as well as the multiconfigurational effects arising from the ionization from different core orbitals, in a spin-pure, black box manner. While non-orthogonal configuration interaction $(\mathrm{NOCI})^{57,58}$ typically requires the appropriate determinants to be chosen on a case-by-case basis, NOCIS is designed to be nearly black-box: for a given molecule and x-ray absorption edge, the set of non-orthogonal determinants is precisely defined. It can also be viewed as a generalization of the static-exchange approximation (STEX) ${ }^{46}$ that moves further by including interactions between different non-orthogonal references. NOCIS provides a dramatic improvement over CIS, and is competitive with or better than other core-excitation methods despite not including dynamic correlation.

In this paper, we present an extension of NOCIS to treat open-shell molecules, specifically doublet radicals. This extension treats all single excitations and their spin complements that can be formed while maintaining the same spin quantum number, which requires the consideration of a greater number of configurations than closed-shell NOCIS. It also maintains the desirable properties of its base, namely in that it remains black-box, size consistent, variational, and spin-pure. In addition to using a separate set of core ionized orbitals for each atom in the desired band, another special set of non-ionized orbitals for each atom in the band should also be included. The design considerations and defining equations for open shell NOCIS are described in Sec. 2.

To test the accuracy of this method, we ran several calculations on both single and mul- 
tiple core orbitals using open-shell NOCIS and compared it to other common core-excitation methods. We found results that were very consistent with our closed-shell comparisons: namely, that NOCIS provides nearly a six-fold improvement on traditional CIS, and that it is competitive with or better than other core-excitation methods despite not containing dynamic correlation. These results are described in Sec. 3, where the effect of the additional references is first assessed, followed by tests against previously reported calculations, as well as tests against the small number of systems for which reported experimental XAS values are available.

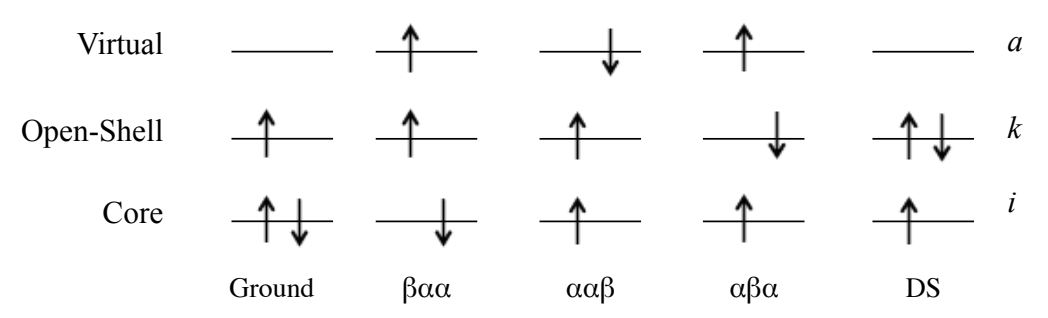

Figure 1: A depiction of different configurations necessary to a core excitation in a doublet radical.

\section{Theory}

When considering a doublet radical excitation, there are several core-excited configurations with $m_{s}=+1 / 2$, which are illustrated in Figure 1 . The first option is the excitation of an alpha electron from the core orbital to an unoccupied virtual orbital, which we have termed a $\beta \alpha \alpha$ excitation, so named for the spins of the electron remaining in the core orbital, openshell orbital, and virtual orbital. The second option is the excitation of a beta electron from the core orbital to an empty virtual orbital, which we have termed a $\alpha \alpha \beta$ excitation. The third option is actually a double excitation: the excitation of a beta electron from the core orbital to an unoccupied virtual followed by a spin-flip in both the open-shell orbital and the virtual orbital. We term this an $\alpha \beta \alpha$ excitation. The final category is the excitation of a beta electron from the core orbital into the open-shell orbital. We term these DS excitations, 
indicating an excitation from a doubly occupied (D) core orbital to a singly-occupied (S) valence orbital. As the $\beta \alpha \alpha, \alpha \alpha \beta$, and $\alpha \beta \alpha$ are all spin-complements of each other, they must all be included in order to ensure that our theory is spin-pure.

These considerations lead us to our open-shell NOCIS wavefunction, shown below:

$\left|\Psi_{N O C I S}\right\rangle=\sum_{i=1}^{R} \sum_{a=1}^{V}(1-\hat{\mathbf{P}})\left(t_{i}^{a}\left[a_{a}^{\dagger} a_{i}\right]+t_{\bar{i}}^{\bar{a}}\left[a_{\bar{a}}^{\dagger} a_{\bar{i}}\right]+t_{\bar{i} k}^{\bar{k}}\left[a_{\bar{k}}^{\dagger} a_{\bar{i}} a_{a}^{\dagger} a_{k}\right]\right)\left|\Phi_{i}\right\rangle+\sum_{i=1}^{R} t_{i}^{k}(1-\hat{\mathbf{P}})\left[a_{\bar{k}}^{\dagger} a_{\bar{i}}\right]\left|\Phi_{i}^{k}\right\rangle$

in which $\left|\Phi_{i}\right\rangle$ is a configuration optimized with a core hole in the $i$ th orbital (there are $R$ relevant core orbitals), but with the core hole filled, also thought of as the ground state configuration, but with core-hole orbitals. $\left|\Phi_{i}^{k}\right\rangle$ is the determinant optimized with a core hole in the $i$ th orbital and the open-shell orbital $k$ doubly occupied but with the core hole filled and the open-shell orbital singly occupied, also thought of as the ground state configuration, but with core-excited-into-open-shell orbitals. As previously, $\hat{\mathbf{P}}$ acts by projecting against the neutral ground state reference. This equation is then solved by the standard generalized eigenvalue problem expression for the core-excited state energies $E_{k}$ and amplitudes $\mathbf{t}_{k}$.

$$
\mathbf{H t}_{k}=\mathbf{S t}_{k} E_{k}
$$

Additionally, as can be seen from the wavefunction, it is important to note that this method has moved beyond the traditional space of single excitations with the inclusion of the $\alpha \beta \alpha$ excitation. This XCIS-type extension ${ }^{59}$ causes open-shell NOCIS to differ from STEX even when core excitations from only a single distinct atom are being considered.

A brief overview of NOCIS is as follows: after a ground-state orbital optimization, a Maximum Overlap Method $(\mathrm{MOM})^{60}$ calculation is done for an excitation from each core orbital of interest (i.e. calculations on core-hole triplet cation configurations of $\hat{a}_{\bar{i}}\left|\phi_{i}\right\rangle$, and the neutral core-hole configurations $\left|\phi_{i}^{k}\right\rangle$ ). The Hamiltonian, $S$ (overlap), and $S^{2}$ (total spin squared) matrices are constructed via a combination of orthogonal methods for interactions between the same core orbital, and NOCI for interactions between different core orbitals. 


\subsection{Restricted Open-Shell Maximum Overlap Method (MOM)}

One of the necessary steps in implementing open-shell NOCIS was the construction of a restricted open-shell MOM method that, while not novel, does not appear to be discussed at much length elsewhere. One goal of using MOM is to prevent excited states from collapsing to lower energy states, which it does during an SCF-like process via the selection of new orbitals that have the largest overlap with the orbitals of the previous iteration. ${ }^{60}$ The 
specific difference for the ROHF case to be addressed here is that, because of the openshell orbital, two separate MOM overlap evaluations must be performed, one for the doubly occupied space and one for the singly occupied space. This is a variation from the usual MOM calculation, which only performs one such evaluation.

\subsection{Diagonal-Block Matrix Elements}

The diagonal-block matrix elements (peach/green in Figure 2) are calculated in the same way as those for closed-shell NOCIS, which is to say computed from Slater-Condon rules or from the appropriate integrals, as in standard CIS or STEX. There is, however, one important difference. With the introduction of the new DS reference state, this renders the aforementioned green areas of these diagonal blocks non-orthogonal to the other determinants in this block, meaning that these elements now need to be calculated with NOCI. We found this new reference to be a necessary addition in terms of accurately describing the potential configurations. This is a further departure from the STEX method.

\subsection{Off-Diagonal Block Matrix Elements}

The multi-reference block matrix elements (blue/green in Figure 2) are generated in the same way as the closed-shell version of NOCIS. Specifically, they are all calculated using NOCI. ${ }^{57,58}$ The only difference is the aforementioned addition of the DS reference, as well as the fact that open-shell NOCIS requires the consideration of more determinants than the closed-shell version, which had only two spin-complements.

\subsection{Computational Implementation}

The code for NOCIS was implemented in a development version of Q-Chem $5.0^{61}$ utilizing the Armadillo linear algebra library. ${ }^{62}$ The NOCI code was a further modified version of the code developed by Sundstrom ${ }^{57}$ and adapted by Oosterbaan et al. ${ }^{54}$ 
The approximate scaling of this code continues to be $N^{4}$. Each NOCI element requires a 2-electron integral build, which is the computational bottleneck. However, it is worth reiterating that NOCIS does not use any large intermediates that require disk storage, an advantage over ADC and EOM-CCSD methods, where the unknowns are as large as fourthrank tensors.

Table 1: A comparison of K-edge deviations from ADC-CVS(2)-x values for molecules with and without the open-shell reference included.

\begin{tabular}{lccc}
\hline \hline Molecule & NOCIS & NOCIS (with DS ref.) & Difference \\
\hline $\mathrm{F}(1 s)$ & 3.73 & 2.07 & -1.67 \\
$\mathrm{OH} \mathrm{O}(1 s)$ & 3.64 & 2.08 & -1.56 \\
$\mathrm{CN} \mathrm{N}(1 s)$ & 0.88 & 0.09 & -0.78 \\
$\mathrm{NO}_{2} \mathrm{O}(1 s)$ & 0.73 & 0.73 & 0.00 \\
\hline \hline
\end{tabular}

\section{Results}

All molecular geometries were obtained from NIST's Computational Chemistry Comparison and Benchmark DataBase, ${ }^{65}$ which uses B3LYP ${ }^{66-68}$ in the Def2-TZVPP basis set, ${ }^{69}$ except for $\mathrm{CH}_{3} \mathrm{~S}$, which was obtained with a calculation in the cc-pCVDZ ${ }^{70}$ basis set using B3LYP. K-edges were selected in all methods by choosing the lowest state with a non-zero oscillator strength. The TDDFT and $\Delta$-DFT calculations were performed with the SRC1R1 functional, ${ }^{71}$ which was specifically designed for core excitations. The TDDFT and CIS calculations were formatted to produce 5 states, and the CVS-ADC(2)-x calculations to produce 3, while the NOCIS calculations produced a large range as dictated by the number of virtual orbitals in the basis set for each atom of interest.

\subsection{Addition of Open-Shell Reference}

The addition of the open-shell reference, the DS excitation configuration(s) that were optimized with a separate SCF calculation, was found to provide a substantial improvement 
Table 2: Comparisons of K-edge calculation errors for several molecules using CIS, $\Delta$-SCF, $\Delta$-DFT, TDDFT, and NOCIS with ADC-CVS(2)-x values, along with the mean signed error (MSE), root-mean-squared error (RMSE), and max error. Calculations were done in the augcc-pCVTZ ${ }^{63,64}$ basis and all results are in $\mathrm{eV}$. The K-edge was defined as the lowest energy transition with non-zero oscillator strength.

\begin{tabular}{|c|c|c|c|c|c|c|}
\hline Molecule & CVS-ADC(2)-x & CIS & $\Delta$-SCF & $\Delta$-DFT & NOCIS & TDDFT \\
\hline $\mathrm{BeH} \mathrm{Be}(1 s)$ & 113.28 & 3.27 & 5.27 & 1.16 & 0.35 & -1.11 \\
\hline $\mathrm{C}_{2} \mathrm{H}_{3} \mathrm{C}(1 s)$ & 281.17 & 7.62 & 3.24 & 4.00 & 1.77 & -0.33 \\
\hline $\mathrm{CCH} \mathrm{C}(1 s)$ & 278.85 & 7.36 & 1.59 & 2.35 & 1.68 & -0.73 \\
\hline $\mathrm{CH}_{3} \mathrm{C}(1 s)$ & 281.09 & 7.40 & 6.02 & 0.38 & 1.55 & -0.47 \\
\hline $\mathrm{H}_{2} \mathrm{COH} \mathrm{C}(1 s)$ & 283.23 & 7.39 & 5.08 & 2.07 & 1.54 & -0.43 \\
\hline $\mathrm{CH}_{3} \mathrm{CH}_{2} \mathrm{O} \mathrm{O}(1 s)$ & 524.96 & 9.87 & 10.97 & 2.57 & 2.25 & -1.41 \\
\hline $\mathrm{HCO} \mathrm{C}(1 s)$ & 284.53 & 7.38 & 0.87 & 1.85 & 1.20 & -0.55 \\
\hline $\mathrm{CN} \mathrm{C}(1 s)$ & 278.96 & 7.01 & 1.45 & 2.20 & 1.12 & -0.59 \\
\hline $\mathrm{CN} \mathrm{N}(1 s)$ & 393.03 & 15.20 & 0.80 & 1.55 & 0.09 & 1.82 \\
\hline $\mathrm{F}(1 s)$ & 675.06 & 10.25 & 2.17 & 2.26 & 2.07 & -3.09 \\
\hline $\mathrm{N}_{2}+\mathrm{N}(1 s)$ & 392.77 & 11.76 & 11.06 & 4.43 & 2.12 & 0.99 \\
\hline $\mathrm{NH}_{2} \mathrm{~N}(1 s)$ & 393.69 & 8.72 & 7.84 & 2.46 & 1.91 & -0.91 \\
\hline $\mathrm{NO} N(1 s)$ & 397.45 & 9.12 & 1.52 & 3.71 & 3.16 & -0.75 \\
\hline $\mathrm{NO} \mathrm{O}(1 s)$ & 530.06 & 14.38 & 1.27 & 4.10 & 2.83 & 1.05 \\
\hline $\mathrm{NO}_{2} \mathrm{~N}(1 s)$ & 400.67 & 9.77 & 2.09 & 2.61 & 0.91 & -0.27 \\
\hline $\mathrm{HCO} \mathrm{O}(1 s)$ & 529.83 & 15.99 & 5.70 & 2.09 & 1.10 & 1.24 \\
\hline $\mathrm{NO}_{2} \mathrm{O}(1 s)$ & 529.04 & 16.26 & 14.04 & 5.37 & 0.73 & 1.41 \\
\hline $\mathrm{O}_{2} \mathrm{CH}_{3} \mathrm{C}(1 s)$ & 286.47 & 9.96 & 2.77 & 0.94 & 0.56 & 0.63 \\
\hline $\mathrm{O}_{2} \mathrm{CH}_{3} \mathrm{O}(1 \mathrm{~s})$ & 527.17 & 10.06 & 9.76 & 5.32 & 1.81 & -0.86 \\
\hline $\mathrm{OH} \mathrm{O}(1 s)$ & 524.84 & 9.73 & 10.35 & 2.48 & 2.08 & -1.73 \\
\hline $\mathrm{H}_{2} \mathrm{COH} \mathrm{O}(1 s)$ & 532.14 & 17.52 & 1.99 & 2.01 & 1.46 & 1.14 \\
\hline $\mathrm{CH}_{3} \mathrm{O} \mathrm{C}(1 s)$ & 283.85 & 14.26 & 5.19 & 1.90 & 1.42 & 1.65 \\
\hline $\mathrm{CH}_{3} \mathrm{O} \mathrm{O}(1 s)$ & 525.14 & 9.87 & 10.80 & 2.56 & 2.10 & -1.40 \\
\hline $\operatorname{Li}(1 s)$ & 56.40 & 2.28 & 2.22 & 6.69 & 1.94 & -0.75 \\
\hline $\mathrm{CH}_{3} \mathrm{~S} \mathrm{C}(1 s)$ & 283.54 & 12.64 & 1.01 & 1.63 & 1.06 & 1.12 \\
\hline $\mathrm{HO}_{2} \mathrm{O}(1 s)$ & 527.21 & 9.93 & 8.79 & 5.54 & 1.81 & -1.10 \\
\hline MSE & & 10.19 & 5.15 & 2.85 & 1.56 & -0.21 \\
\hline RMSE & & 10.84 & 6.49 & 3.24 & 1.71 & 1.21 \\
\hline Max & & 17.52 & 14.04 & 6.69 & 3.16 & 3.09 \\
\hline
\end{tabular}




\subsection{Comparison with Common Core-Excitation Methods}

Following our previous basis set assessment, we performed our comparison calculations in the aug-cc-pCVTZ basis, which was determined to provide the best balance of error reduction and efficiency. This can be seen in Table 2. Due to a relative lack of experimental spectra for small open-shell molecules, we compared all our calculations to CVS-ADC(2)-x to make an inital assessment of accuracy. Of course, this requires the presumption that CVS-ADC(2)$\mathrm{x}$ is a suitable reference. The accuracy of this comparison to experimental data will be discussed in the following section.

As with closed-shell molecules, we compared NOCIS to CIS, $\Delta$-SCF, $\Delta$-DFT, and TDDFT. $\Delta$-DFT and TDDFT were performed using the SRC1-R1 functional, ${ }^{71}$ which is tailored to performing core-excitation calculations in TDDFT. All calculations other than NOCIS were performed using UHF and are thus not spin-pure, and it was found that the difference when using ROHF for the $\Delta$ methods was negligible.

Our results are largely consistent with our previous closed-shell results. ${ }^{79}$ As expected, 
Table 3: A comparison of the minimum and maximum errors from the CVS-ADC(2)-x values for CIS, $\triangle$-SCF, $\triangle$-DFT, NOCIS, and TDDFT.

\begin{tabular}{lcccccc}
\hline \hline & & CIS & $\Delta$-SCF & $\Delta$-DFT & NOCIS & TDDFT \\
\hline $\mathrm{C}(1 s)$ & $\min$ & 7.01 & 0.87 & 0.38 & 0.56 & -0.33 \\
& $\max$ & 14.26 & 6.02 & 4.00 & 1.77 & 1.65 \\
$\mathrm{~N}(1 s)$ & $\min$ & 8.72 & 0.80 & 1.55 & 0.09 & -0.27 \\
& $\max$ & 15.20 & 11.06 & 4.43 & 3.16 & 1.82 \\
$\mathrm{O}(1 s)$ & $\min$ & 9.73 & 1.27 & 2.01 & 0.73 & -0.86 \\
& $\max$ & 17.52 & 10.04 & 5.54 & 2.83 & -1.73 \\
\hline \hline
\end{tabular}

Table 4: A comparison of errors from experimental values of CVS-ADC(2)-x, NOCIS, and TDDFT.

\begin{tabular}{lcccc}
\hline \hline Molecule & Expt. & ADC & NOCIS & TDDFT \\
\hline $\mathrm{NO}_{2} \mathrm{~N}(1 s)$ & $401.04^{72}$ & -0.37 & 0.54 & -0.65 \\
$\mathrm{NO}_{2} \mathrm{O}(1 s)$ & $530.32^{72}$ & -1.28 & -0.55 & 0.13 \\
$\mathrm{NO} \mathrm{N}(1 s)$ & $399.70^{73}$ & -0.80 & 0.91 & -3.00 \\
$\mathrm{NO} \mathrm{O}(1 s)$ & $532.70^{73}$ & -1.76 & -0.19 & -1.58 \\
$\mathrm{~F}(1 s)$ & $676.5^{74}$ & -1.44 & 0.63 & -4.52 \\
$\mathrm{Li}(1 s)$ & $57.39^{75}$ & -0.98 & 0.96 & -1.74 \\
$\mathrm{CH}_{3} \mathrm{~S} \mathrm{C}(1 s)$ & $286.7^{76}$ & -3.16 & -2.10 & -2.04 \\
$\mathrm{OH} \mathrm{O}(1 s)$ & $525.80^{77}$ & -0.96 & 1.12 & -2.69 \\
$\mathrm{HO}_{2} \mathrm{O}(1 s)$ & $528.60^{77}$ & -1.39 & 0.42 & -2.49 \\
$\mathrm{CH}_{3} \mathrm{C}(1 s)$ & $281.35^{78}$ & -0.26 & 1.29 & -0.73 \\
\hline MSE & & -1.24 & 0.34 & -1.93 \\
RMSE & & 1.46 & 1.01 & 2.31 \\
Max & & -3.16 & -2.10 & -4.52 \\
\hline \hline
\end{tabular}


the CIS method performs very poorly due to its lack of orbital relaxation and correlation, with a RMS error of $10.84 \mathrm{eV}$. The $\triangle$-SCF method, which includes orbital relaxation but not dynamic correlation, is a large improvement on CIS methods, with a RMS error of 6.49 $\mathrm{eV}$. The $\Delta$-DFT methods, which include both orbital relaxation and dynamic correlation, perform even better, as expected, with a RMS error of $3.24 \mathrm{eV}$. The TDDFT methods perform extremely well, with an RMS error of $1.21 \mathrm{eV}$. This high level of accuracy is in large part due to the correct selection of functional, and it shows the importance of correlation in obtaining an accurate core-excitation energy. However, TDDFT is extremely functional dependent, and performing it with other functionals produces results that vary considerably in accuracy.

The NOCIS calculations, which include orbital relaxation and static correlation but not dynamic correlation, perform very consistently with previous closed-shell results, with a RMS error of $1.71 \mathrm{eV}$. This result trend upholds our previous conclusion that while dynamic correlation is important (and becomes more so with open-shell molecules), the inclusion of orbital relaxation has a far greater impact on improving the accuracy of results. In fact, the NOCIS results provide more than a six-fold improvement on the CIS results.

A further element to look at in these methods is whether the errors appear to be random or systematic, which we have explored in Table 3. CIS and $\Delta$-SCF appear highly unsystematic, with an average error spread of around 7.2 and $8.1 \mathrm{eV}$, respectively. $\Delta$-DFT performs better, with an average error spread of around $3.3 \mathrm{eV}$. NOCIS and TDDFT perform the most systematically, with average error spreads of 2.1 and 1.7 respectively. Overall, these results provide encouraging signs that NOCIS is a relatively systematic method, with a systematic level of error, furthering its desirability.

\subsection{Comparison to Experimental Data}

In the interest of comparing across a broad set of molecules, we first benchmarked our NOCIS results to CVS-ADC(2)-x. There are, however, a limited amount of experimental spectra 
for doublet radicals available, and comparison to those energies produced some interesting results, which are summarized in Table 4.

Across different ADC methods, it appears that most forms of ADC overestimate core excitation energies, but CVS-ADC(2)-x appears to tend toward underestimation. ${ }^{45}$ This is consistent with our comparisons here, and this data set is large enough to reasonably draw the conclusion that it is possible that our reported NOCIS results for molecules without an experimental spectra that are higher than the ADC values will be slightly closer to experimental values than their reported error here indicates. It is very encouraging that NOCIS out performs both CVS-ADC(2)-x and TDDFT with the tuned SRC1-R1 functional on these radicals.

\subsection{Oscillator Strengths and Spectra}

For a core excitation calculation to produce spectra, it needs to be able to calculate oscillator strengths in addition to excitation energies and multiplicities. These oscillator strengths are then used to construct the spectra. Experimentally, oscillator strengths are quite difficult to determine, and are used only in relative terms between species. Thus, there is not a significant amount of experimental data available to which to compare our calculations.

Table 5: A comparison of calculated oscillator strengths for CIS, NOCIS, and ADC-CVS(2)-x

\begin{tabular}{lccc}
\hline \hline Molecule & CIS & NOCIS & ADC \\
\hline $\mathrm{NO}_{2} \mathrm{~N}(1 s)$ & 0.0464 & 0.0411 & 0.0191 \\
$\mathrm{NO}_{2} \mathrm{O}(1 s)$ & 0.0625 & 0.0470 & 0.0322 \\
$\mathrm{NO}(1 s)$ & 0.0112 & 0.0463 & 0.0613 \\
$\mathrm{NO} \mathrm{O}(1 s)$ & 0.0218 & 0.0319 & 0.0367 \\
$\mathrm{~F}(1 s)$ & 0.0680 & 0.0571 & 0.0510 \\
$\mathrm{CH}_{3} \mathrm{~S} \mathrm{C}(1 s)$ & 0.00198 & 0.000667 & 0.000623 \\
$\mathrm{OH} \mathrm{O}(1 s)$ & 0.0652 & 0.0515 & 0.0449 \\
$\mathrm{HO}_{2} \mathrm{O}(1 s)$ & 0.0642 & 0.0581 & 0.0418 \\
$\mathrm{CH}_{3} \mathrm{C}(1 s)$ & 0.0584 & 0.0428 & 0.0357 \\
\hline \hline
\end{tabular}

As an alternative, Table 5 shows a comparison of oscillator strengths between CIS, NOCIS, and ADC-CVS(2)-x. Taking ADC as our reference point, we see that NOCIS oscillator 
2

3

4

5

6

7

8

10

11

strengths in every case improves on the CIS results in the direction of ADC, often substantially. This is encouraging for the direct use of NOCIS to simulate experimental spectra.

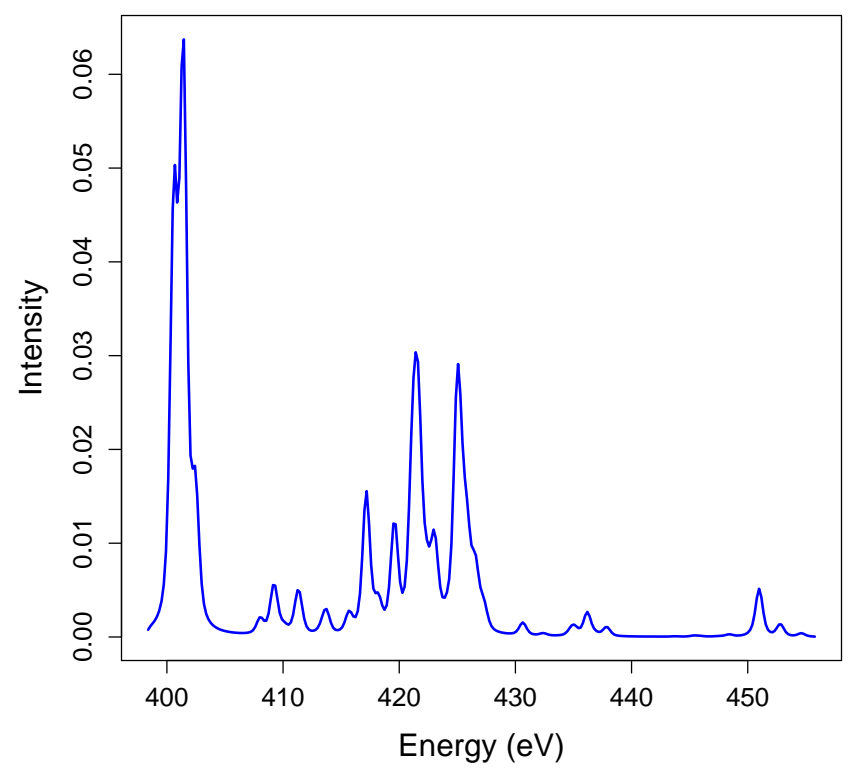

Figure 3: The NOCIS simulation of the NO N K-edge spectrum. This spectrum has a Gaussian broadening of .25 eV and a Lorentzian broadening of .2 eV, consistent with previous work. ${ }^{76}$ See Table 6 for peak centers and assignments in comparison to experiment, ADC and CIS.

To test the accuracy of NOCIS in simulating experimental spectra, we plotted the NOCIS data for the NO N K-edge, as shown in Figure 3. The spectrum is in quite good qualitative agreement with the experimental spectrum presented in Reference 73. For a more quantitative assessment, we also identified the first few spectral peaks for for CIS, NOCIS, and ADC-CVS(2)-x, and compared those to the experimental assignments from Ref. 73. This comparison is presented in Table 6.

Referring to Table 6, it is clear from the identified transitions that CIS performs very poorly. While CIS does correctly associate the K-edge energy with the $2 \mathrm{p} \pi^{*}$ transition, it did not adequately identify any of the other transitions. This is likely a consequence of increasing spin-contamination for the higher states, as well as the lack of orbital relaxation. By contrast, 
Table 6: A comparison to experiment of transition assignments and energies for the first few peaks in the NO N K-edge spectrum for CIS, NOCIS, and ADC.

\begin{tabular}{cccccccc}
\hline \hline \multicolumn{2}{c}{ Experiment } & \multicolumn{2}{c}{ CIS } & \multicolumn{2}{c}{ NOCIS } & \multicolumn{2}{c}{ ADC } \\
Energy & Transition & Energy & Transition & Energy & Transition & Energy & Transition \\
\hline 399.7 & $2 \mathrm{p} \pi^{*}$ & 406.6 & $2 \mathrm{p} \pi^{*}$ & 400.6 & $2 \mathrm{p} \pi^{*}$ & 397.4 & $2 \mathrm{p} \pi^{*}$ \\
404.0 & $2 \mathrm{p} \sigma^{*}$ & - & - & - & - & 404.7 & double \\
406.6 & $3 \mathrm{~s} \sigma$ & - & - & 408.03 & $3 \mathrm{~s} \sigma(\mathrm{O})$ & 407.17 & $3 \mathrm{~s} \sigma(\mathrm{O})$ \\
407.6 & $3 \mathrm{~s} \sigma$ & 422.07 & $3 \mathrm{~s} \sigma(\mathrm{N})$ & 409.1 & $3 \mathrm{~s} \sigma(\mathrm{N})$ & 407.4 & $3 \mathrm{~s} \sigma(\mathrm{N})$ \\
& $3 \mathrm{p} \pi$ & - & - & 409.3 & $3 \mathrm{p} \pi$ & 407.6 & $3 \mathrm{p} \pi$ \\
409.0 & $3 \mathrm{p}$ & 408.7 & $3 \mathrm{p}$ & 410.8 & $3 \mathrm{p}$ & 409.2 & $3 \mathrm{p}$ \\
\hline \hline
\end{tabular}

NOCIS provides a substantial improvement, with peaks that for the most part qualitatively replicate the experimental spectrum and correctly identify the transitions. Despite its lack of dynamic correlation, NOCOS is competitive with ADC-CVS(2)-x, which provides a higher degree of accuracy on the energies, at the cost of substantially longer computation time.

There is one peak to which this conclusion does not apply, however, We observe that peak 2 of Table 6 (404.0 eV, experimentally), is present only in the ADC spectrum, but not in the CIS or NOCIS spectra. This, we believe, is because this transition has been misidentified by the authors of Reference 73 . We believe that, instead of a transition to the $2 p \sigma^{*}$ orbital, it is in fact primarily a double excitation that involves both a valence excitation from the singlyoccupied orbital and an excitation from the core orbital into the $2 p \pi^{*}$ orbital, as identified by the ADC data. This new identification as a double excitation clearly explains why the transition is absent in the NOCIS and CIS spectra, which operate only in the space of single excitations. In sum, it appears that the NOCIS energies and oscillator strengths are enough to generate a spectral profile that can be directly and reliably compared with experimental data for single-excitation XAS.

\section{Conclusions}

This paper describes the extension of the NOCIS method to treat doublet open-shell molecules. This method compares favorably to all current methods of calculating core excitations, and 


\section{Acknowledgements}

KJO thanks the members of the Head-Gordon group for fruitful discussions. This work was supported by the Director, Office of Science, Office of Basic Energy Sciences of the US 
Department of Energy under contract No. DE-AC02-05CH11231.

\section{References}

(1) Phaniendra, A.; Jestadi, D. B.; Periyasamy, L. Free Radicals: Properties, Sources, Targets, and Their Implication in Various Diseases. Indian J. Clin. Biochem. 2015, 30, 11-26.

(2) Giles, N. M.; Giles, G. I.; Jacob, C. Multiple roles of cysteine in biocatalysis. Biochem. Biophys. Res. Commun. 2003, 300, 1-4.

(3) Marinov, N. M.; Castaldi, M. J.; Melius, C. F.; Tsang, W. Aromatic and Polycyclic Aromatic Hydrocarbon Formation in a Premixed Propane Flame. Combust. Sci. Technol. 1997, 128, 295-342.

(4) Herbst, E. The chemistry of interstellar space. Chem. Soc. Rev. 2001, 30, 168-176.

(5) Keller, C.; Anicich, V.; Cravens, T. Model of Titans ionosphere with detailed hydrocarbon ion chemistry. Planet. Space Sci. 1998, 46, 1157-1174.

(6) Anicich, V. G.; McEwan, M. J. Ion-molecule chemistry in Titan's ionosphere. Planet. Space Sci. 1997, 45, 897-921.

(7) McEwan, M. J.; Scott, G. B.; Anicich, V. G. Ion-molecule reactions relevant to Titan's ionosphere. Int. J. Mass Spectrom. Ion Processes 1998, 172, 209-219.

(8) Laffon, C.; Lacombe, S.; Bournel, F.; Parent, P. Radiation effects in water ice: A near-edge x-ray absorption fine structure study. J. Chem. Phys. 2006, 125, 204714.

(9) Laffon, C.; Lasne, J.; Bournel, F.; Schulte, K.; Lacombe, S.; Parent, P. Photochemistry of carbon monoxide and methanol in water and nitric acid hydrate ices: A NEXAFS study. Phys. Chem. Chem. Phys. 2010, 12, 10865. 
(10) Martin-Diaconescu, V.; Kennepohl, P. Sulfur K-edge XAS as a probe of sulfur-centered radical intermediates. J. Am. Chem. Soc. 2007, 129, 3034-3035.

(11) Wang, Z.; Popolan-Vaida, D. M.; Chen, B.; Moshammer, K.; Mohamed, S. Y.; Wang, H.; Sioud, S.; Raji, M. A.; Kohse-Höinghaus, K.; Hansen, N.; Dagaut, P.; Leone, S. R.; Sarathy, S. M. Unraveling the structure and chemical mechanisms of highly oxygenated intermediates in oxidation of organic compounds. Proc. Natl. A. Sci. 2017, 114, 13102-13107.

(12) Alagia, M.; Bodo, E.; Decleva, P.; Falcinelli, S.; Ponzi, A.; Richter, R.; Stranges, S. The soft X-ray absorption spectrum of the allyl free radical. Phys. Chem. Chem. Phys. 2013, 15, 1310-1318.

(13) Yang, Z.; Schnorr, K.; Bhattacherjee, A.; Lefebvre, P.-L.; Epshtein, M.; Xue, T.; Stanton, J. F.; Leone, S. R. Electron-Withdrawing Effects in the Photodissociation of CH 2 ICl To Form CH 2 Cl Radical, Simultaneously Viewed Through the Carbon K and Chlorine L 2,3 X-ray Edges. J. Am. Chem. Soc. 2018, 140, 13360-13366.

(14) Laffon, C.; Lasne, J.; Bournel, F.; Schulte, K.; Lacombe, S.; Parent, P. Photochemistry of carbon monoxide and methanol in water and nitric acid hydrate ices: A NEXAFS study. Phys. Chem. Chem. Phys. 2010, 12, 10865.

(15) Lochan, R. C.; Head-Gordon, M. Orbital-optimized opposite-spin scaled second-order correlation: An economical method to improve the description of open-shell molecules. J. Chem. Phys. 2007, 126, 164101.

(16) Byrd, E. F.; Sherrill, C. D.; Head-Gordon, M. The theoretical prediction of molecular radical species: A systematic study of equilibrium geometries and harmonic vibrational frequencies. J. Phys. Chem. A 2001, 105, 9736-9747.

(17) Farnell, L.; Pople, J. A.; Radom, L. Structural predictions for open-shell systems: a comparative assessment of ab initio procedures. J. Phys. Chem. 1983, 87, 79-82. 
(18) Tozer, D. J.; Handy, N. C.; Amos, R. D.; Pople, J. A.; Nobes, R. H.; Xie, Y.; Schaefer, H. F. Theory and applications of spin-restricted open-shell Møller-Plesset theory. Mol. Phys. 1993, 79, 777-793.

(19) Wong, M. W.; Radom, L. Radical Addition to Alkenes : A Theoretical Perspective. J. Phys. Chem. A 1998, 102, 2237-2245.

(20) Seeger, R.; Pople, J. A. Selfconsistent molecular orbital methods. XVIII. Constraints and stability in HartreeFock theory. J. Chem. Phys. 1977, 66, 3045-3050.

(21) Lee, J.; Head-Gordon, M. Distinguishing artificial and essential symmetry breaking in a single determinant: approach and application to the C 60 , C 36 , and C 20 fullerenes. Phys. Chem. Chem. Phys. 2019, 21, 4763-4778.

(22) Johnson, B. G.; Gonzales, C. A.; Gill, P. M.; Pople, J. A. A density functional study of the simplest hydrogen abstraction reaction. Effect of self-interaction correction. Chem. Phys. Lett. 1994, 221, 100-108.

(23) Kristyán, S.; Pulay, P. Can (semi)local density functional theory account for the London dispersion forces? Chem. Phys. Lett. 1994, 229, 175-180.

(24) Handy, N. C.; Pople, J. A.; Head-Gordon, M.; Raghavachari, K.; Trucks, G. W. Sizeconsistent Brueckner theory limited to double substitutions. Chem. Phys. Lett. 1989, 164, 185-192.

(25) Krylov, A. I.; Sherrill, C. D.; Byrd, E. F. C.; Head-Gordon, M. Size-consistent wave functions for nondynamical correlation energy: The valence active space optimized orbital coupled-cluster doubles model. J. Chem. Phys. 1998, 109, 10669.

(26) Pople, J. A.; Gill, P. M.; Johnson, B. G. KohnSham density-functional theory within a finite basis set. Chem. Phys. Lett. 1992, 199, 557-560. 
(30) Ekström, U.; Norman, P. X-ray absorption spectra from the resonant-convergent firstorder polarization propagator approach. Phys. Rev. A 2006, 74, 042722.

(31) Ekström, U.; Norman, P.; Carravetta, V.; Ågren, H. Polarization Propagator for X-Ray Spectra. Phys. Rev. Lett. 2006, 97, 143001.

(32) Besley, N. A.; Gilbert, A. T. B.; Gill, P. M. W. Self-consistent-field calculations of core excited states. J. Chem. Phys. 2009, 130, 124308.

(33) Krylov, A. I. Equation-of-Motion Coupled-Cluster Methods for Open-Shell and Electronically Excited Species: The Hitchhiker's Guide to Fock Space. Annu. Rev. Phys. Chem. 2008, 59, 433-462.

(34) Monkhorst, H. J. Calculation of properties with the coupled-cluster method. Int. J. Quantum Chem. 2009, 12, 421-432.

(35) Sadybekov, A.; Krylov, A. I. Coupled-cluster based approach for core-level states in condensed phase: Theory and application to different protonated forms of aqueous glycine. J. Chem. Phys. 2017, 147, 014107.

(36) Coriani, S.; Christiansen, O.; Fransson, T.; Norman, P. Coupled-cluster response theory 
for near-edge x-ray-absorption fine structure of atoms and molecules. Phys. Rev. A 2012, 85, 022507 .

(37) Fransson, T.; Coriani, S.; Christiansen, O.; Norman, P. Carbon X-ray absorption spectra of fluoroethenes and acetone: A study at the coupled cluster, density functional, and static-exchange levels of theory. J. Chem. Phys. 2013, 138, 124311.

(38) List, N. H.; Coriani, S.; Kongsted, J.; Christiansen, O. Lanczos-driven coupledcluster damped linear response theory for molecules in polarizable environments. J. Chem. Phys. 2014, 141, 244107.

(39) Peng, B.; Lestrange, P. J.; Goings, J. J.; Caricato, M.; Li, X. Energy-Specific Equationof-Motion Coupled-Cluster Methods for High-Energy Excited States: Application to K -edge X-ray Absorption Spectroscopy. J. Chem. Theory Comput. 2015, 11, 4146-4153.

(40) Cederbaum, L. S.; Domcke, W.; Schirmer, J.; von Niessen, W. Many-Body Effects in Valence and Core Photoionization of Molecules. Phys. Scr. 1980, 21, 481-491.

(41) Barth, A.; Cederbaum, L. S. Many-body theory of core-valence excitations. Phys. Rev. A 1981, 23, 1038-1061.

(42) Barth, A.; Schirmer, J. Theoretical core-level excitation spectra of N 2 and CO by a new polarisation propagator method. J. Phys. B: At. Mol. Phys. 1985, 18, 867-885.

(43) Plekan, O.; Feyer, V.; Richter, R.; Coreno, M.; de Simone, M.; Prince, K.; Trofimov, A.; Gromov, E.; Zaytseva, I.; Schirmer, J. A theoretical and experimental study of the near edge X-ray absorption fine structure (NEXAFS) and X-ray photoelectron spectra (XPS) of nucleobases: Thymine and adenine. Chem. Phys. 2008, 347, 360-375.

(44) Feyer, V.; Plekan, O.; Richter, R.; Coreno, M.; Vall-llosera, G.; Prince, K. C.; Trofimov, A. B.; Zaytseva, I. L.; Moskovskaya, T. E.; Gromov, E. V.; Schirmer, J. Tau- 
tomerism in Cytosine and Uracil: An Experimental and Theoretical Core Level Spectroscopic Study. J. Phys. Chem. A 2009, 113, 5736-5742.

(45) Wenzel, J.; Holzer, A.; Wormit, M.; Dreuw, A. Analysis and comparison of CVS-ADC approaches up to third order for the calculation of core-excited states. J. Chem. Phys. 2015, 142, 214104.

(46) Ågren, H.; Carravetta, V.; Vahtras, O.; Pettersson, L. G. M. Direct SCF direct staticexchange calculations of electronic spectra. Theor. Chem. Acc. 1997, 97, 14-40.

(47) Foresman, J. B.; Head-Gordon, M.; Pople, J. A.; Frisch, M. J. Toward a systematic molecular orbital theory for excited states. J. Phys. Chem. 1992, 96, 135-149.

(48) Head-Gordon, M.; Grana, A. M.; Maurice, D.; White, C. A. Analysis of Electronic Transitions as the Difference of Electron Attachment and Detachment Densities. J. Phys. Chem. 1995, 99, 14261-14270.

(49) Wang, F.; Ziegler *, T. Excitation energies of some d 1 systems calculated using timedependent density functional theory: an implementation of open-shell TDDFT theory for doubletdoublet excitations. Mol. Phys. 2004, 102, 2585-2595.

(50) Nooijen, M.; Bartlett, R. J. Description of coreexcitation spectra by the openshell electronattachment equationofmotion coupled cluster method. J. Chem. Phys. 1995, 102, 6735-6756.

(51) Roemelt, M.; Maganas, D.; DeBeer, S.; Neese, F. A combined DFT and restricted open-shell configuration interaction method including spin-orbit coupling: Application to transition metal L-edge X-ray absorption spectroscopy. J. Chem. Phys. 2013, 138, 204101.

(52) Wenzel, J.; Wormit, M.; Dreuw, A. Calculating X-ray Absorption Spectra of Open-Shell 
Molecules with the Unrestricted Algebraic-Diagrammatic Construction Scheme for the Polarization Propagator. J. Chem. Theory Comput. 2014, 10, 4583-4598.

(53) Coriani, S.; Koch, H. Communication: X-ray absorption spectra and core-ionization potentials within a core-valence separated coupled cluster framework. J. Chem. Phys. 2015, 143, 181103.

(54) Oosterbaan, K. J.; White, A. F.; Head-Gordon, M. Non-orthogonal configuration interaction with single substitutions for the calculation of core-excited states. J. Chem. Phys. 2018, 149, 044116.

(55) Ehlert, C.; Klamroth, T. The quest for best suited references for configuration interaction singles calculations of core excited states. J. Comput. Chem. 2017, 38, 116-126.

(56) Ehlert, C.; Gühr, M.; Saalfrank, P. An efficient first principles method for molecular pump-probe NEXAFS spectra: Application to thymine and azobenzene. J. Chem. Phys. 2018, 149, 144112.

(57) Sundstrom, E. J.; Head-Gordon, M. Non-orthogonal configuration interaction for the calculation of multielectron excited states. J. Chem. Phys. 2014, 140, 114103.

(58) Thom, A. J. W.; Head-Gordon, M. HartreeFock solutions as a quasidiabatic basis for nonorthogonal configuration interaction. J. Chem. Phys. 2009, 131, 124113.

(59) Maurice, D.; Head-Gordon, M. On the Nature of Electronic Transitions in Radicals: An Extended Single Excitation Configuration Interaction Method. J. Phys. Chem. 1996, $100,6131-6137$.

(60) Gilbert, A. T. B.; Besley, N. A.; Gill, P. M. W. Self-Consistent Field Calculations of Excited States Using the Maximum Overlap Method (MOM) . J. Phys. Chem. A 2008, 112, 13164-13171. 
(61) Shao, Y.; Gan, Z.; Epifanovsky, E.; Gilbert, A. T.; Wormit, M.; Kussmann, J.; Lange, A. W.; Behn, A.; Deng, J.; Feng, X.; Ghosh, D.; Goldey, M.; Horn, P. R.; Jacobson, L. D.; Kaliman, I.; Khaliullin, R. Z.; Kuś, T.; Landau, A.; Liu, J.; Proynov, E. I.; Rhee, Y. M.; Richard, R. M.; Rohrdanz, M. A.; Steele, R. P.; Sundstrom, E. J.; Woodcock, H. L.; Zimmerman, P. M.; Zuev, D.; Albrecht, B.; Alguire, E.; Austin, B.; Beran, G. J. O.; Bernard, Y. A.; Berquist, E.; Brandhorst, K.; Bravaya, K. B.; Brown, S. T.; Casanova, D.; Chang, C.-M.; Chen, Y.; Chien, S. H.; Closser, K. D.; Crittenden, D. L.; Diedenhofen, M.; DiStasio, R. A.; Do, H.; Dutoi, A. D.; Edgar, R. G.; Fatehi, S.; Fusti-Molnar, L.; Ghysels, A.; Golubeva-Zadorozhnaya, A.; Gomes, J.; Hanson-Heine, M. W.; Harbach, P. H.; Hauser, A. W.; Hohenstein, E. G.; Holden, Z. C.; Jagau, T.-C.; Ji, H.; Kaduk, B.; Khistyaev, K.; Kim, J.; Kim, J.; King, R. A.; Klunzinger, P.; Kosenkov, D.; Kowalczyk, T.; Krauter, C. M.; Lao, K. U.; Laurent, A. D.; Lawler, K. V.; Levchenko, S. V.; Lin, C. Y.; Liu, F.; Livshits, E.; Lochan, R. C.; Luenser, A.; Manohar, P.; Manzer, S. F.; Mao, S.-P.; Mardirossian, N.; Marenich, A. V.; Maurer, S. A.; Mayhall, N. J.; Neuscamman, E.; Oana, C. M.; Olivares-Amaya, R.; O’Neill, D. P.; Parkhill, J. A.; Perrine, T. M.; Peverati, R.; Prociuk, A.; Rehn, D. R.; Rosta, E.; Russ, N. J.; Sharada, S. M.; Sharma, S.; Small, D. W.; Sodt, A.; Stein, T.; Stück, D.; Su, Y.-C.; Thom, A. J.; Tsuchimochi, T.; Vanovschi, V.; Vogt, L.; Vydrov, O.; Wang, T.; Watson, M. A.; Wenzel, J.; White, A.; Williams, C. F.; Yang, J.; Yeganeh, S.; Yost, S. R.; You, Z.-Q.; Zhang, I. Y.; Zhang, X.; Zhao, Y.; Brooks, B. R.; Chan, G. K.; Chipman, D. M.; Cramer, C. J.; Goddard, W. A.; Gordon, M. S.; Hehre, W. J.; Klamt, A.; Schaefer, H. F.; Schmidt, M. W.; Sherrill, C. D.; Truhlar, D. G.; Warshel, A.; Xu, X.; Aspuru-Guzik, A.; Baer, R.; Bell, A. T.; Besley, N. A.; Chai, J.-D.; Dreuw, A.; Dunietz, B. D.; Furlani, T. R.; Gwaltney, S. R.; Hsu, C.-P.; Jung, Y.; Kong, J.; Lambrecht, D. S.; Liang, W.; Ochsenfeld, C.; Rassolov, V. A.; Slipchenko, L. V.; Subotnik, J. E.; Van Voorhis, T.; Herbert, J. M.; Krylov, A. I.; Gill, P. M.; Head-Gordon, M. Advances in molecular quantum chemistry contained in 
the Q-Chem 4 program package. Mol. Phys. 2015, 113, 184-215.

(62) Sanderson, C.; Curtin, R. Armadillo: a template-based C++ library for linear algebra. Journal of Open Source Software 2016, 1, 26.

(63) Kendall, R. A.; Dunning, T. H.; Harrison, R. J. Electron affinities of the firstrow atoms revisited. Systematic basis sets and wave functions. J. Chem. Phys. 1992, 96, 67966806.

(64) Woon, D. E.; Dunning, T. H. Gaussian basis sets for use in correlated molecular calculations. III. The atoms aluminum through argon. J. Chem. Phys. 1993, 98, 1358-1371.

(65) NIST, CCCBDB calculated geometries. 2018; https://cccbdb.nist.gov/geom1x. asp.

(66) Becke, A. D. Densityfunctional thermochemistry. III. The role of exact exchange. J. Chem. Phys. 1993, 98, 5648-5652.

(67) Lee, C.; Yang, W.; Parr, R. G. Development of the Colle-Salvetti correlation-energy formula into a functional of the electron density. Phys. Rev. B 1988, 37, 785-789.

(68) Vosko, S. H.; Wilk, L.; Nusair, M. Accurate spin-dependent electron liquid correlation energies for local spin density calculations: a critical analysis. Can. J. Phys. 1980, 58, 1200-1211.

(69) Weigend, F.; Ahlrichs, R. Balanced basis sets of split valence, triple zeta valence and quadruple zeta valence quality for H to Rn: Design and assessment of accuracy. Phys. Chem. Chem. Phys. 2005, 7, 3297.

(70) Dunning, T. H. Gaussian basis sets for use in correlated molecular calculations. I. The atoms boron through neon and hydrogen. J. Chem. Phys. 1989, 90, 1007-1023. 
(71) Besley, N. A.; Peach, M. J. G.; Tozer, D. J. Time-dependent density functional theory calculations of near-edge X-ray absorption fine structure with short-range corrected functionals. Phys. Chem. Chem. Phys. 2009, 11, 10350.

(72) Zhang, W.; Sze, K.; Brion, C.; Tong, X.; Li, J. Inner-shell electron energy loss spectra of NO2 at high resolution: Comparison with multichannel quantum defect calculations of dipole oscillator strengths and transition energies. Chem. Phys. 1990, 140, 265-279.

(73) Wight, G.; Brion, C. K-shell excitations in NO and O2 by $2.5 \mathrm{keV}$ electron impact. J. Electron Spectrosc. Relat. Phenom. 1974, 4, 313-325.

(74) Svensson, S.; Karlsson, L.; Mårtensson, N.; Baltzer, P.; Wannberg, B. Electron spectroscopy observation of the $1 \mathrm{~s} 2 \mathrm{~s} 22 \mathrm{p} 6(2 \mathrm{~S})$ state in atomic fluorine by means of electron impact excitation of the hf molecule. J. Electron Spectrosc. Relat. Phenom. 1990, 50, $\mathrm{C} 1-\mathrm{C} 7$.

(75) Ziem, P.; Stolterfoht, N.; Bruch, R. Autoionization spectra of Li I and Li II excited by $\mathrm{H}+$ and He + impact. J. Phys. B: At. Mol. Phys. 1975, 8, L480-L484.

(76) Schnorr, K.; Bhattacherjee, A.; Oosterbaan, K. J.; Delcey, M. G.; Yang, Z.; Xue, T.; Attar, A. R.; Chatterley, A. S.; Head-Gordon, M.; Leone, S. R.; Gessner, O. Tracing the 267 nm-Induced Radical Formation in Dimethyl Disulfide Using Time-Resolved X-ray Absorption Spectroscopy. J. Phys. Chem. Let. 2019, 10, 1382-1387.

(77) Lacombe, S.; Bournel, F.; Laffon, C.; Parent, P. Radical Photochemistry in OxygenLoaded Ices. Ang. Chem. 2006, 118, 4265-4269.

(78) Alagia, M.; Lavollée, M.; Richter, R.; Ekström, U.; Carravetta, V.; Stranges, D.; Brunetti, B.; Stranges, S. Probing the potential energy surface by high-resolution x-ray absorption spectroscopy: The umbrella motion of the core-excited C H 3 free radical. Phys. Rev. A 2007, 76, 022509. 
(79) Oosterbaan, K. J.; White, A. F.; Head-Gordon, M. Erratum: Non-orthogonal configuration interaction with single substitutions for the calculation of core-excited states [J. Chem. Phys. 149, 044116 (2018)]. J. Chem. Phys. 2018, 149, 139901.

(80) Yost, S. R.; Head-Gordon, M. Size consistent formulations of the perturb-thendiagonalize Møller-Plesset perturbation theory correction to non-orthogonal configuration interaction. J. Chem. Phys. 2016, 145, 054105.

(81) Yost, S. R.; Head-Gordon, M. Efficient Implementation of NOCI-MP2 Using the Resolution of the Identity Approximation with Application to Charged Dimers and Long CC Bonds in Ethane Derivatives. J. Chem. Theory Comput. 2018, 14, 4791-4805. 

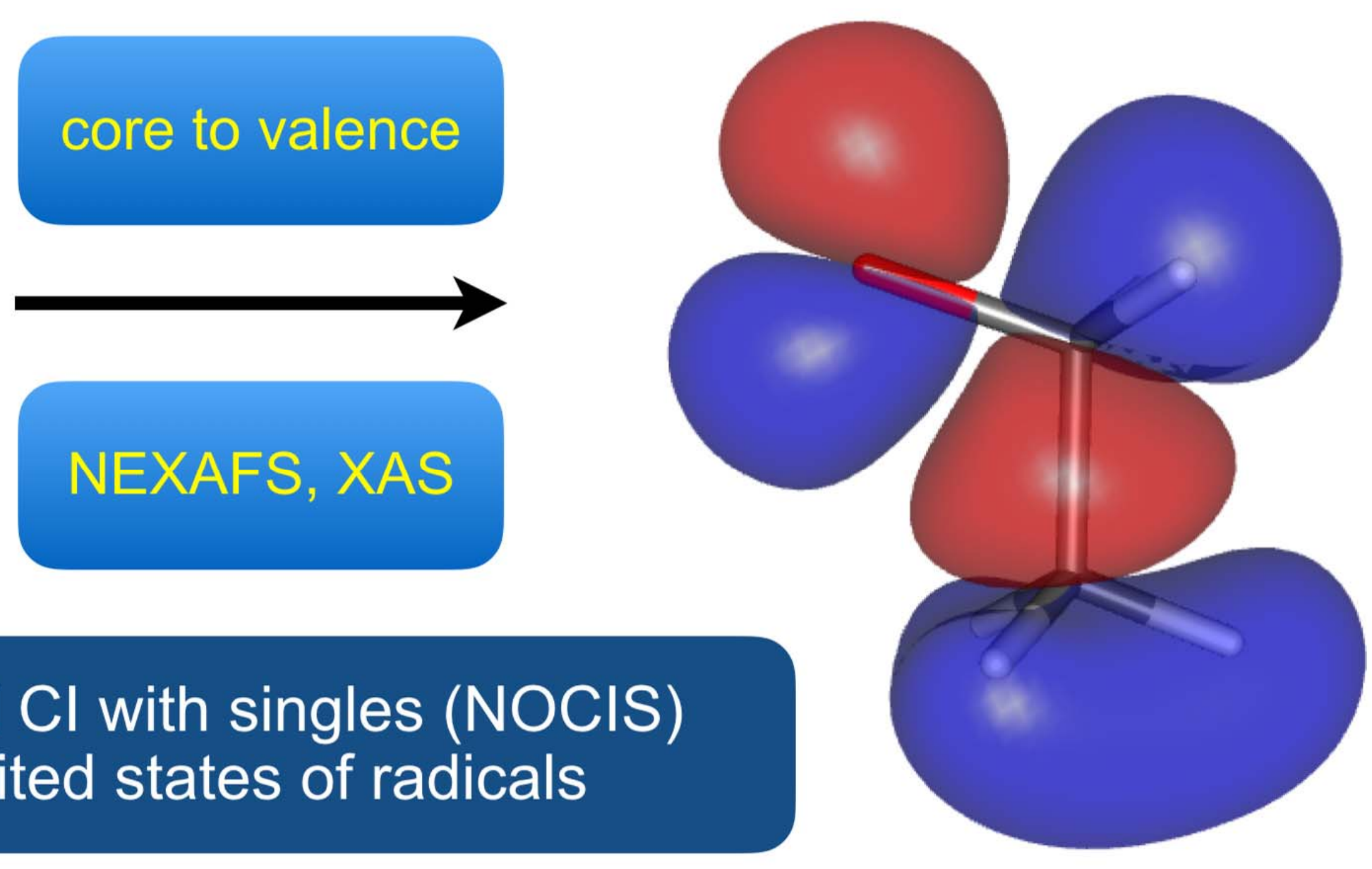

\section{Non-orthogonal $\mathrm{Cl}$ with singles (NOCIS)} for core-excited states of radicals 